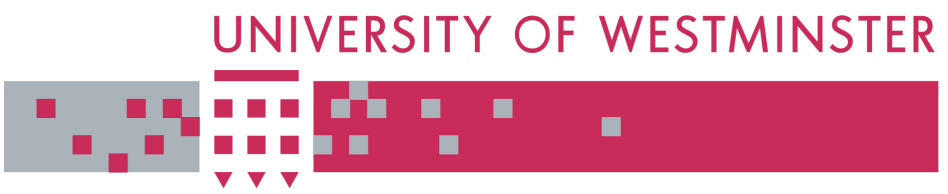

WestminsterResearch

http://www.wmin.ac.uk/westminsterresearch

\title{
A low complexity DSP driven analog impairment mitigation scheme for low-IF GNSS receivers
}

\section{Alper Ucar \\ Ediz Cetin \\ Izzet Kale}

School of Informatics, University of Westminster

Copyright (C) [2008] IEEE. Reprinted from the proceedings of the IEEE/ION Position, Location and Navigation Symposium 2008: May 6-8, 2008, Hyatt Regency Hotel, Monterey, California. IEEE, Los Alamitos, USA, pp. 865-870. ISBN 9781424415366.

This material is posted here with permission of the IEEE. Such permission of the IEEE does not in any way imply IEEE endorsement of any of the University of Westminster's products or services. Personal use of this material is permitted. However, permission to reprint/republish this material for advertising or promotional purposes or for creating new collective works for resale or redistribution to servers or lists, or to reuse any copyrighted component of this work in other works must be obtained from the IEEE. By choosing to view this document, you agree to all provisions of the copyright laws protecting it.

The WestminsterResearch online digital archive at the University of Westminster aims to make the research output of the University available to a wider audience. Copyright and Moral Rights remain with the authors and/or copyright owners.

Users are permitted to download and/or print one copy for non-commercial private study or research. Further distribution and any use of material from within this archive for profit-making enterprises or for commercial gain is strictly forbidden.

Whilst further distribution of specific materials from within this archive is forbidden, you may freely distribute the URL of the University of Westminster Eprints (http://www.wmin.ac.uk/westminsterresearch).

In case of abuse or copyright appearing without permission e-mail wattsn@wmin.ac.uk. 


\title{
A Low Complexity DSP Driven Analog Impairment Mitigation Scheme for Low-IF GNSS Receivers
}

\author{
Alper Ucar, Ediz Cetin, and Izzet Kale \\ Applied DSP and VLSI Research Group \\ University of Westminster, United Kingdom
}

\begin{abstract}
Due to the increasing demands for location based services within the wireless mass-market; there has been relentless pressure to reduce both the chip area and power dissipation of the user terminal. Low-IF receivers combine the advantages of superheterodyne and direct-conversion architectures offering a highly integrated solution while avoiding the issues associated with DC offsets and flicker noise. The main drawback of the low-IF architecture is its limited image rejection due to analog impairments. In this paper, the sources of the impairments are analyzed for a low-IF receiver operating at the GPS/Galileo L1 band together with a novel low-complexity solution to compensate for them in the DSP domain is proposed. For processing the combined GPS/Galileo L1 signal, a signal simulator we call GNSScope has been developed together with a low-IF receiver model to analyze the influence of the analog impairments The idea behind our proposed novel adaptive compensator which estimates and compensates for the imbalances and mismatches is that in the absence of these mismatches no correlation exists between the desired and the image channels, which is not the case when impairments are present. Results show that through the deployment of the proposed approach, image-rejection performance can be enhanced by $75 \mathrm{~dB}$. This enhancement in the image-rejection performance subsequently results in relaxed analog front-end specifications leading to high levels of integration making it possible for highly integrated software-defined Global Navigation Satellite Systems (GNSS) receiver to be realistically and economically designed and implemented.
\end{abstract}

\section{INTRODUCTION}

With the development of the European navigation system Galileo and the modernization plans for GPS, it is apparent that the civilian navigation signals will be in multiple bands within the next decade offering various applications from roadside assistance to medical services. Therefore, the next generation navigation user terminal will have to be a multimode versatile wideband receiver in order to cope with multiple bands and modulation schemes. In such a context, the flexibility offered by Software Defined Radio (SDR) is expected to become the dominant technology in GNSS receiver development.

The zero-IF architecture has been considered as a suitable candidate to realize SDR platforms [1]-[3] which utilize one mixer stage to convert the RF signal directly to and from the baseband without the need for external filters. This enables the integration of the receiver on a single monolithic die.
However, zero-IF receivers suffer from corruption of the signal by the DC offsets and flicker noise. One way to avoid the problems associated with DC offsets and flicker noise is to perform digitization at the IF. The low-IF architecture [4]-[6], shown in Fig. 1, is a promising approach due to its insensitivity to parasitic effects such as DC offset and flicker noise, however, it demands higher image rejection which hinders its widespread use in commercial applications.

L1 band, centered as $1575.46 \mathrm{MHz}$, currently contains the GPS signal composed of the civilian C/A and the encrypted $\mathrm{P}(\mathrm{Y})$ code. With the deployment of Galileo, three other channels, L1-A, L1-B, and L1-C, will be available. Galileo introduces Binary Offset Carrier (BOC) modulation, coherent adaptive subcarrier modulation, and pilot signals [7], which will offer superior performance in signal tracking, while providing compatibility and interoperability with other signals in the L1 band. As illustrated in Fig. 2, the GNSS L1 has a null-to-null bandwidth of $32.73 \mathrm{MHz}$ [8]. The next-generation L1 band receiver should be able to receive and demodulate this wideband for the enhanced operation. Compared to conventional narrowband GPS receivers designed for the C/A code reception, a wideband low-IF L1 receiver will have an IF of at least $65 \mathrm{MHz}$.

In this paper, an analysis of the analog impairments leading to limited image rejection is given and a low-complexity solution to enhance the image rejection is proposed. Simulations are carried out with the low-IF receiver model for GPS and Galileo L1 signals. The rest of the paper is organized as follows: Section II introduces analog impairments associated with the quadrature downconverter and the complex filter. Section III presents the proposed novel approach to compensate for them in the digital domain. Section IV presents the simulation results and finally Section V is the concluding remarks. 


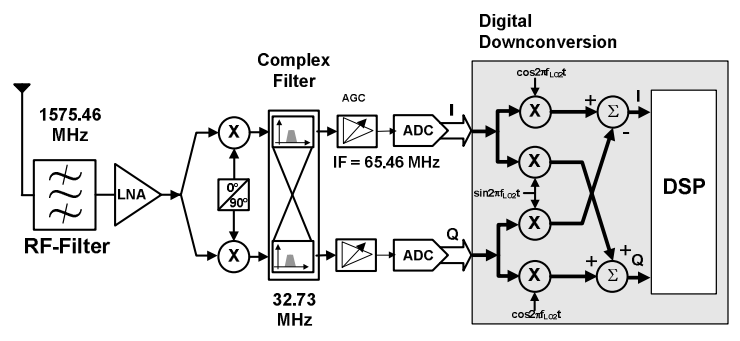

Figure 1. Low-IF Receiver Architecture for L1 Band

\section{ANALOG IMPAIRMENT MODEL}

\section{A. I/Q Imbalance}

Low-IF receivers utilize quadrature downconversion to translate incoming signal from Radio-Frequency (RF) to an Intermediate-Frequency (IF) near DC. The quadrature downconverter suffers from a number of analog impairments namely the gain and phase imbalances in the in-phase (I) and quadrature (Q) paths of the receiver [9]-[11]. Sources of these imbalances are the RF splitter used to divide incoming RF signal between the I and Q paths, the differences in the length of the two RF paths, and the imperfections in the quadrature $90^{\circ}$ phase splitter. An analytical model of a quadrate downconverter with the gain and phase imbalances and complex filter mismatch is illustrated in Fig. 3. In this model, gain and phase imbalances are characterized by two parameters: the gain mismatch, and the phase orthogonality mismatch, between the I and Q branches.

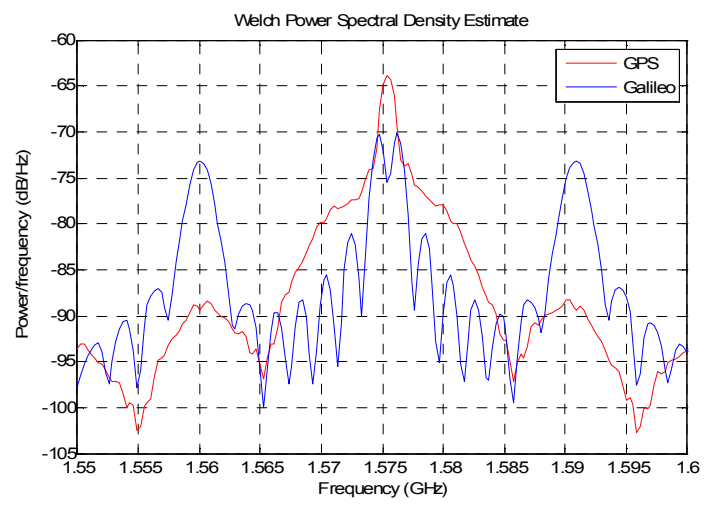

Figure 2. GPS and Galileo Signals generetaded by GNSScope

The following is an outline analysis to demonstrate the effects of analog impairments on receiver's performance. The incoming RF signal, $s(t)$, consists of the desired signal at $\omega_{R F}$ and the image at $\omega_{I M}=\omega_{R F}-2 \omega_{I F}$. Hence, $s(t)$ can be written as

$$
s(t)=\mathrm{RF}(\mathrm{t})+\mathrm{IM}(\mathrm{t})=\Re\left\{u(t) e^{j \omega_{R F} t}\right\}+\Re\left\{i(t) e^{j \omega_{M} t}\right\}
$$

where $\operatorname{IM}(\mathrm{t})$ is the image of the desired signal $\mathrm{RF}(\mathrm{t}), \omega_{I F}$ is the intermediate frequency, $u(t)$ and $i(t)$ are the complex envelopes of the received signals at $\omega_{R F}$ and $\omega_{I M}$, respectively. When I/Q imbalance is not present LO signal can be expressed as

$$
I_{L O}+j Q_{L O}=\cos \left(\omega_{L O} t\right)-j \sin \left(\omega_{L O} t\right)=2 e^{-j \omega_{L O} t} .
$$

In this ideal case, there will be no corruption in the downconverted signal constellation which can be written as

$$
s_{I F}(t)=s(t) 2 e^{-j \omega_{L O} t} .
$$

However, due to $\mathrm{I} / \mathrm{Q}$ imbalances the erroneous complex LO signal, $\tilde{I}_{L O}+\tilde{Q}_{L O}$, incorporates gain and phase mismatches

$$
\tilde{I}_{L O}+\tilde{Q}_{L O}=\frac{1}{2} e^{j \omega_{L O} t}\left(g_{1} e^{j \frac{\varphi_{\varepsilon}}{2}}-g_{2} e^{j \frac{\varphi_{\varepsilon}}{2}}\right)+e^{-j \omega_{L O} t}\left(g_{1} e^{-j \frac{\varphi_{\varepsilon}}{2}}+g_{2} e^{j \frac{\varphi_{\varepsilon}}{2}}\right)
$$

where $g_{1}=(1+0.5 \alpha \varepsilon)$ and $g_{2}=(1-0.5 \alpha \varepsilon)$ The output of the frequency downconversion, ignoring the high frequency components, is

$$
\begin{array}{r}
x(t)=u(t)\left(g_{1} e^{-j \frac{\varphi_{\varepsilon}}{2}}+g_{2} e^{j \frac{\varphi_{\varepsilon}}{2}}\right) e^{j \omega_{I_{F} t}}+u^{*}(t)\left(g_{1} e^{j \frac{\varphi_{\varepsilon}}{2}}-g_{2} e^{-j \frac{\varphi_{\varepsilon}}{2}}\right) e^{-j \omega_{I F} t}+ \\
i(t)\left(g_{1} e^{-j \frac{\varphi_{\varepsilon}}{2}}+g_{2} e^{j \frac{\varphi_{\varepsilon}}{2}}\right) e^{-j \omega_{F F} t}+i^{*}(t)\left(g_{1} e^{j \frac{\varphi_{\varepsilon}}{2}}-g_{2} e^{-j \frac{\varphi_{\varepsilon}}{2}}\right) e^{j \omega_{F} t}
\end{array}
$$

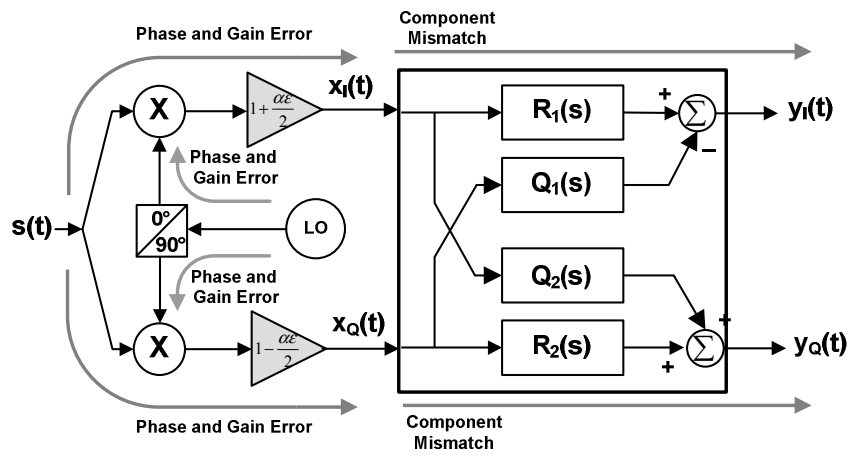

Figure 3. Analog impairment model in low-if receivers

The corresponding frequency domain representation of the process is illustrated in Fig. 4. As one can observe from the signal spectra in Fig. 4(b), the desired signal is translated to IF along with interference from the image signal at $-\omega_{I M}$. The desired signal is then retrieved by a complex filter, which will be discussed in the next section. 

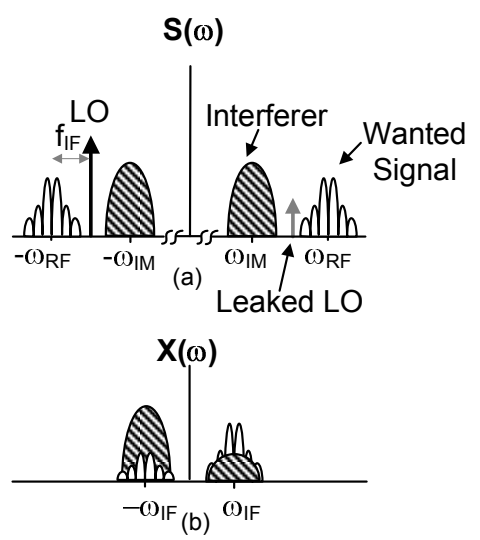

Figure 4. The desired, image, and LO signals (a) before frequency conversion, (b) after frequency conversion.

\section{B. Complex Filter Mismatch}

Complex filters in low-IF receivers employ cross coupling between I and Q paths to achieve a frequency response that is not symmetrical around DC in order to eliminate the undesired components in the signal spectra as illustrated in Fig. 4(b). A complex filter can be realized either by frequency shifting a lowpass filter into complex bandpass or by employing a direct synthesis with complex summers, amplifiers, and integrators for deriving the complex transfer function [12].

For a first order lowpass filter of bandwidth $\omega_{b}$, applying the frequency translation from DC to $\omega_{I F}\left(s \rightarrow s-j \omega_{I F}\right)$ results in the complex transfer function

$$
H(s)=\frac{1}{s-\left(\omega_{b}+j \omega_{I F}\right)}
$$

The result of (6) is a single complex pole which cannot be realized with a real filter. In order to realize the complex pole, two matched filters can be used with cross-coupling as is shown in Fig. 5 [4].

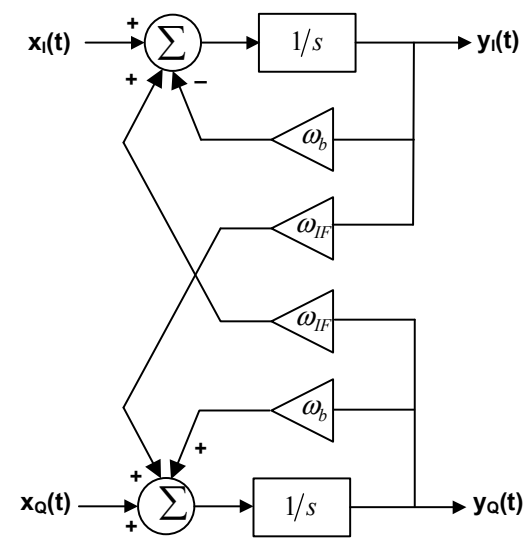

Figure 5. Realization of a single complex pole with two real filters
In analog IC implementations, perfect matching of components is not achievable. Component mismatch in a complex filter can be modeled by perturbing poles and zeros from their nominal values. For a single complex pole as illustrated in Fig. 5, (7) and (8) give approximate expressions for the pole position mismatch.

$$
\begin{gathered}
\omega_{b 1}=\omega_{b}\left(1+\Delta \omega_{b}\right) \\
\omega_{b 2}=\omega_{b}\left(1-\Delta \omega_{b}\right) \\
\omega_{I F 1}=\omega_{I F}\left(1+\Delta \omega_{I F}\right) \\
\omega_{I F 2}=\omega_{I F}\left(1-\Delta \omega_{I F}\right)
\end{gathered}
$$

The expressions for the complex pole outputs can be written as

$$
\begin{aligned}
& y_{\mathrm{I}}(t)=\frac{1}{s}\left(x_{\mathrm{I}}(t)+\omega_{b 1} y_{\mathrm{I}}(t)-\omega_{I F 1} y_{\mathrm{Q}}(t)\right) \\
& y_{\mathrm{Q}}(t)=\frac{1}{s}\left(x_{\mathrm{Q}}(t)+\omega_{b 2} y_{\mathrm{Q}}(t)-\omega_{I F 2} y_{\mathrm{I}}(t)\right)
\end{aligned}
$$

which illustrates the effect of mirroring form desired to undesired frequencies and vice versa. Rearranging the terms in (9) yields

$$
y_{\mathrm{I}}(t)=\frac{x_{\mathrm{I}}(t)\left(\omega_{I F}-\omega_{I F} \Delta \omega_{I F}\right)+x_{\mathrm{Q}}(t)\left(s-\omega_{b}-\omega_{b} \Delta \omega_{b}\right)}{s^{2}-2 \omega_{b} s+\left(\omega_{b}^{2}-\left(\omega_{b} \Delta \omega_{b}\right)^{2}+\omega_{I F}{ }^{2}-\left(\omega_{I F} \Delta \omega_{I F}\right)^{2}\right.}
$$

and

$$
y_{\mathrm{Q}}(t)=\frac{x_{\mathrm{I}}(t)\left(s-\omega_{b}-\omega_{b} \Delta \omega_{b}\right)-x_{\mathrm{Q}}(t)\left(\omega_{I F}-\omega_{I F} \Delta \omega_{I F}\right)}{s^{2}-2 \omega_{b} s+\left(\omega_{b}^{2}-\left(\omega_{b} \Delta \omega_{b}\right)^{2}+\omega_{I F}{ }^{2}-\left(\omega_{I F} \Delta \omega_{I F}\right)^{2}\right.}
$$

Due to the perturbations introduced, the desired signal at $\omega_{I F}$ will be filtered by an average transfer function

$$
H_{\mathrm{AVG}}(s)=\frac{1}{2}\left\{R_{1}(s)+R_{2}(s)\right\}+j\left\{Q_{1}(s)+Q_{2}(s)\right\}
$$

while component mismatch will introduce an undesired filter

$$
\Delta H_{\mathrm{DIFF}}(s)=\frac{1}{2}\left\{R_{1}(s)-R_{2}(s)\right\}+j\left\{Q_{1}(s)-Q_{2}(s)\right\} .
$$

where

$$
\begin{aligned}
& R_{1}(s)=\frac{s-\omega_{b 2}}{s^{2}-\left(\omega_{b 1}+\omega_{b 2}\right) s+\omega_{b 1} \omega_{b 2+} \omega_{I F 1} \omega_{I F 1}} \\
& R_{2}(s)=\frac{s-\omega_{b 1}}{s^{2}-\left(\omega_{b 1}+\omega_{b 2}\right) s+\omega_{b 1} \omega_{b 2+} \omega_{I F 1} \omega_{I F 1}} \\
& Q_{1}(s)=\frac{\omega_{I F 1}}{s^{2}-\left(\omega_{b 1}+\omega_{b 2}\right) s+\omega_{b 1} \omega_{b 2+} \omega_{I F 1} \omega_{I F 2}}
\end{aligned}
$$


and

$$
Q_{2}(s)=\frac{\omega_{I F 2}}{s^{2}-\left(\omega_{b 1}+\omega_{b 2}\right) s+\omega_{b 1} \omega_{b 2+} \omega_{I F 1} \omega_{I F 2}}
$$

Referring to Fig. 3, the output of the complex filter in can be written as [13]

$$
Y(s)=H_{\mathrm{AVG}}(s) X(s)+\Delta H_{\mathrm{DIFF}}(s) X^{*}(s)
$$

where $X^{*}(s)$ is the complex conjugate of $X(s)$. Incorporating gain and phase errors due to $\mathrm{I} / \mathrm{Q}$ imbalance into (18) results in

$$
Y(s)=H_{\mathrm{AVG}}^{\prime}(s) S_{I F}(s)+\Delta H_{\mathrm{DIFF}}^{\prime}(s) S_{I F}^{*}(s)
$$

where

$$
\begin{gathered}
H_{\mathrm{AVG}}^{\prime}(s)= \\
\frac{1}{2} e^{-j \frac{\varphi \varepsilon}{2}}\left\{H_{\mathrm{AVG}}(s)\left(g_{1}\left(1+e^{j \varphi \varepsilon}\right)\right)+\right. \\
\left.\Delta H_{D I F F}(s)\left(g_{2}+g_{1} e^{j \varphi \varepsilon}\right)\right\}
\end{gathered}
$$

and

$$
\begin{aligned}
\Delta H_{\mathrm{DIFF}}^{\prime}(s)= & \frac{1}{2} e^{-j \frac{\varphi \varepsilon}{2}}\left\{H_{\mathrm{DIFF}}(s)\left(g_{2}\left(1-e^{j \varphi \varepsilon}\right)\right)+\right. \\
& \left.H_{\mathrm{AVG}}(s)\left(g_{1}+g_{2} e^{j \varphi \varepsilon}\right)\right\} .
\end{aligned}
$$

Fig. 6 shows the signal constellation defined in (19) ignoring the high frequency components.

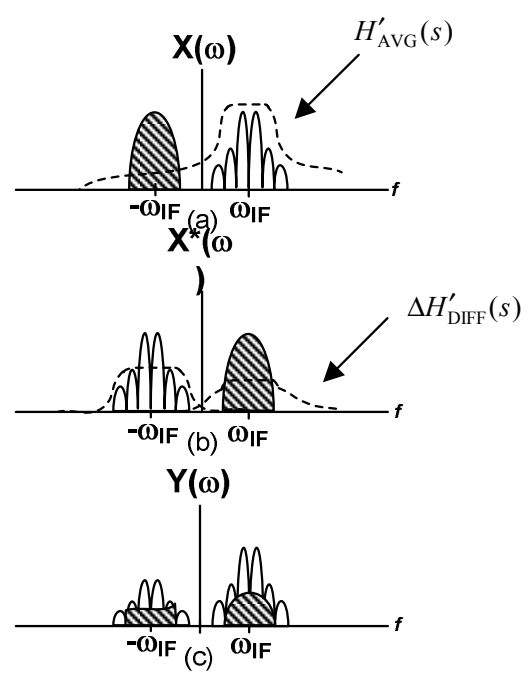

Figure 6. Illustration of (20), (21), and (18)

\section{Image Rejection Ratio}

A measure of the influence of the effects of the analog impairments can be performed by Image Rejection Ratio (IRR). IRR is defined as the attenuation of the desired signal due to image leakage. There are three sources of interference in the signal constellation in Fig. 6. These are image leakage through $\Delta H_{\mathrm{DIFF}}^{\prime}\left(j \omega_{I F}\right)$ and the desired signal leakage through $\Delta H_{\mathrm{DIFF}}^{\prime}\left(-j \omega_{I F}\right)$ and $H_{\mathrm{AVG}}^{\prime}\left(-j \omega_{I F}\right)$. Hence, the image rejection ratio (IRR) can be defined as

$$
\operatorname{IRR}=\frac{\left|H_{\mathrm{AVG}}^{\prime}\left(j \omega_{I F}\right)\right|^{2}}{\left|\Delta H_{\mathrm{DIFF}}^{\prime}\left(j \omega_{I F}\right)\right|^{2}+\left|\Delta H_{\mathrm{DIFF}}^{\prime}\left(-j \omega_{I F}\right)\right|^{2}+\left|H_{\mathrm{AVG}}^{\prime}\left(-j \omega_{I F}\right)\right|^{2}}
$$

The values of $\left|\Delta H_{\mathrm{DIFF}}^{\prime}\left(-j \omega_{I F}\right)\right|^{2}$ and $\left|H_{\mathrm{AVG}}^{\prime}\left(-j \omega_{I F}\right)\right|^{2}$ determine the out-of-band interference. The in-band IRR can be written as

$$
\operatorname{IRR}_{\text {in-band }}=\frac{\left|H_{\mathrm{AVG}}^{\prime}\left(j \omega_{I F}\right)\right|^{2}}{\left|\Delta H_{\mathrm{DIFF}}^{\prime}\left(j \omega_{I F}\right)\right|^{2}}
$$

\section{Digital IMPAIRMENT Mitigation BlOCK}

Our proposed solution to mitigate analog impairments is the Digital Impairment Mitigation Block (DIMB) [11],[14]. which is based on blind source separation by adaptive algorithms. DIBM processes the digitized low-IF signals and estimates and compensates the mismatches in real-time domain. The idea behind the proposed approach is that in the absence of the mismatches no correlation exists between the desired and the image signals.

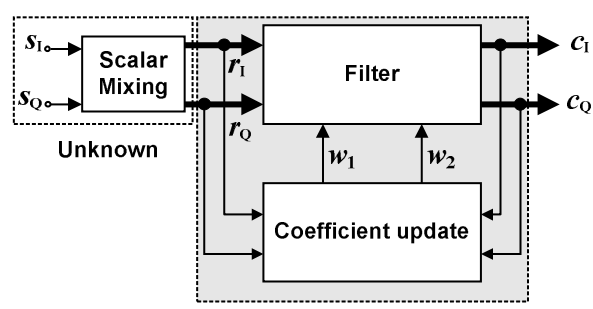

Figure 7. Structure of the DIMB

In the proposed approach the filter block consists of 2-taps, $w 1$ and $w_{2}$. Output signals $c_{\mathrm{I}}$ and $c_{\mathrm{Q}}$ can be expressed as a function of transmitted signals $s_{\mathrm{I}}$ and $s_{\mathrm{Q}}$ as:

$$
\begin{aligned}
& c_{I}(k)=\left(1-w_{1} h_{2}\right) s_{I}(k)+\left(h_{1}-w_{1}\right) s_{Q}(k) \\
& c_{Q}(k)=\left(h_{2}-w_{2}\right) s_{I}(k)+\left(1-w_{2} h_{2}\right) s_{Q}(k)
\end{aligned}
$$

where $h_{1}$ and $h_{2}$ can are functions of gain and phase errors and component mismatches which depend on the complex filter type and order. When the filters converge, i.e. $w_{1}=h_{1}$ and $w_{2}=h_{2}$, the source estimates become:

$$
\begin{aligned}
& c_{I}(k)=\left(1-h_{1} h_{2}\right) s_{I}(k) \\
& c_{Q}(k)=\left(1-h_{1} h_{2}\right) s_{Q}(k)
\end{aligned}
$$


As can be observed from (25) the influence of the analog impairments has been removed. However, both I and Q channels are scaled by $\left(1-h_{1} h_{2}\right)$ which can be safely ignored. The coefficient update can be done with any adaptive algorithm depending on the desired performance with leastmean-square and recursive-least-squares algorithms being the most obvious ones resulting in different convergence speeds and computational complexities.

\section{SIMLATION RESULTS}

In order to study, analyze and evaluate the performance of our approach, a low-IF transmission reception model is developed as illustrated in Fig. 8. In this model, a GPS/Galileo signal generator for L1 band is used for the desired signal while an M-PSK signal of bandwidth $32 \mathrm{MHz}$ is used for the image centered at $1444.54 \mathrm{MHz}$. Quadrature downconverter and the fourth order complex Butterworth filter of bandwidth $32.73 \mathrm{MHz}$ incorporates the analog impairments. The outputs of the simulator are the complex baseband signals of the desired GNSS L1 and the image which are fed into the DIMB.

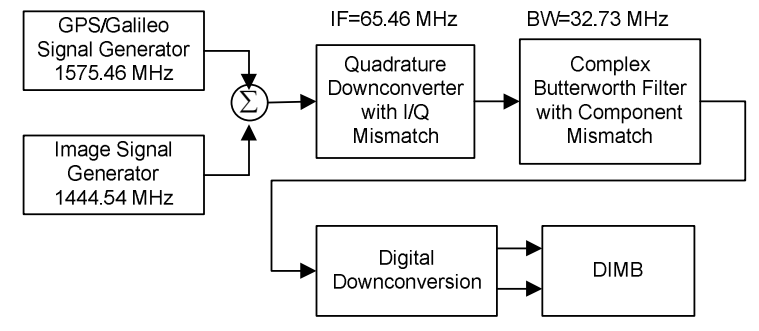

Figure 8. Low-if transmission and reception model

The output of the complex filter is shown in Fig. 9. As seen in figure, for a fixed gain and phase error, the value of $\left|\Delta H_{\mathrm{DIFF}}^{\prime}\left(-j \omega_{I F}\right)\right|$ increases with the component mismatch factor of the complex filter. In contrast, I/Q imbalances contribute to $\left|\Delta H_{\mathrm{DIFF}}^{\prime}\left(j \omega_{I F}\right)\right|$. The IRR as a function of gain and phase errors and component mismatches is plotted in Fig. 10 while the output of the DIMB is given in Fig. 11. After compensation, the image rejection is improved up to $75 \mathrm{~dB}$. It has previously reported that an image rejection of 20 to $25 \mathrm{~dB}$ is adequate for the reception of the $\mathrm{C} / \mathrm{A}$ code [6],[15] since the IF can be conveniently selected below $8 \mathrm{MHz}$. For this choice the image lies in the L1 band and mainly consists of thermal noise. However, this is not the case when dealing with the wideband L1 signal. Furthermore, Galileo signals for E5 and E6 will introduce wider bands than L1 hence the next-generation lowIF GNSS receivers will demand dramatic enhancements in image rejection compared to that of the conventional GPS receiver.
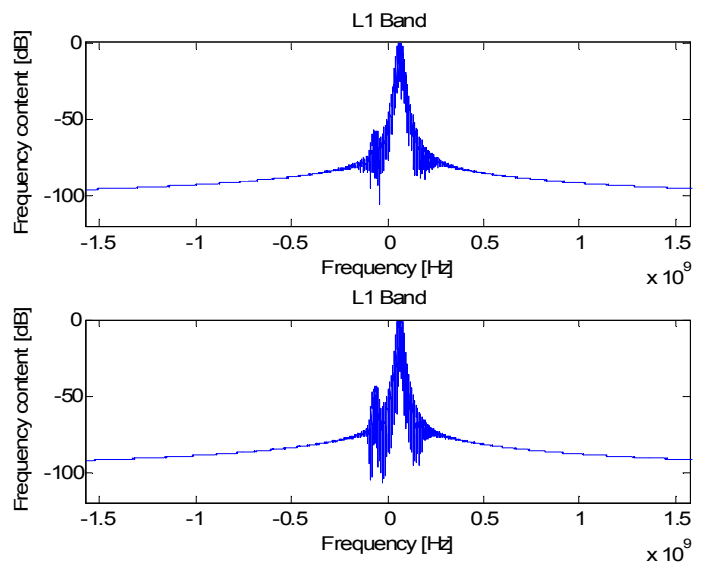

Figure 9. Output of the forth order complex Butterworth filter for a fixed gain error of $\% 1$ and phase error of 0.2 radians with (a) $\% 0.5$ component mismatch (b) $\% 1$ component mismatch...

\section{CONCLUDING REMARKS}

Due to the increasing demands for wireless services, user terminal is required to be compact, low-power and low-cost. Receiver architectures that offer integration on a single monolithic die suffer from I/Q and component mismatches. In this paper, the sources for these impairments are analyzed and a low-complexity solution to compensate for them is proposed. Simulation results show that by using the DIMB, image-rejection-performance can be enhanced by $75 \mathrm{~dB}$. This results in relaxed analog front-end specification enabling high levels of integration and leading a path to single chip GNSS receiver. 

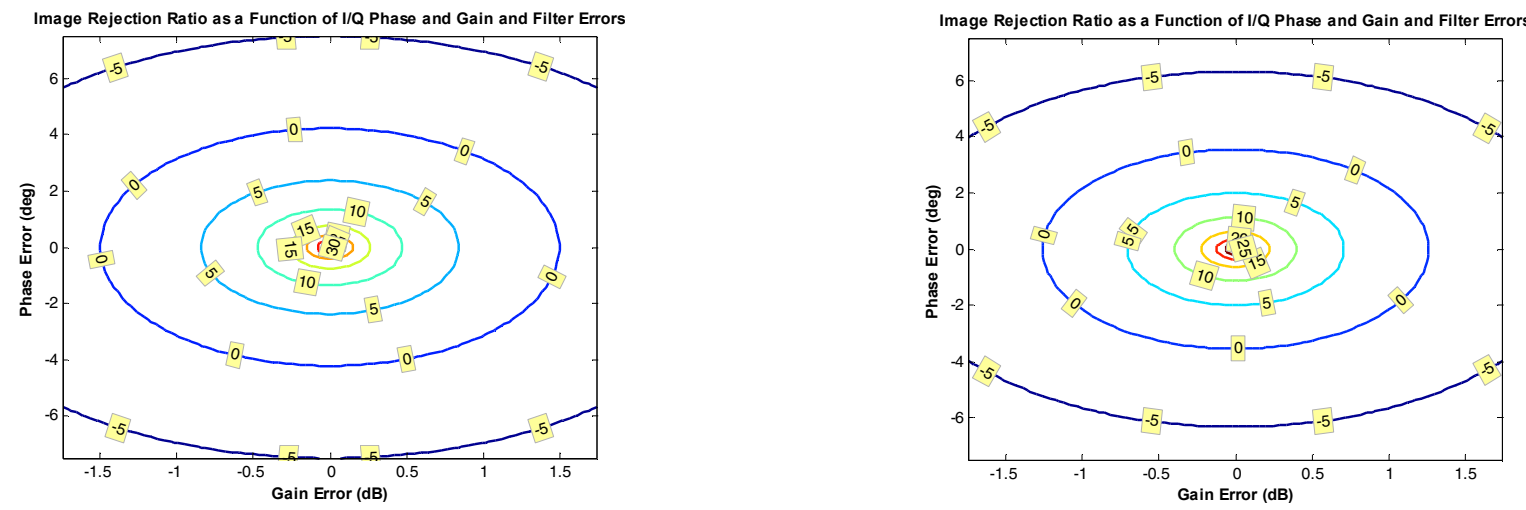

Figure 10. IRR as a function of I/Q imbaalnces and complex filter component mismatch (a) $0.5 \%$ (b) $1 \%$.
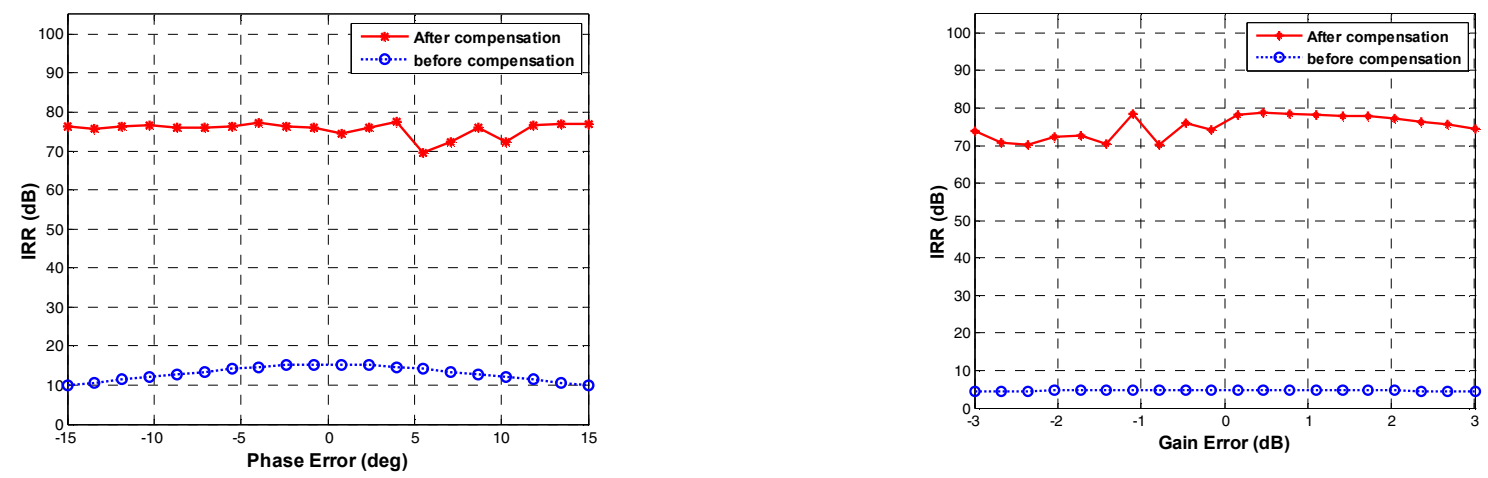

Figure 11. IRR before and after compensation for varying (a) phase and (b) gain errors with complex filter component mismatch of $1 \%$.

\section{REFERENCES}

[1] Abidi, A. A., "The Path to the Software-Defined Radio Receiver," Solid-State Circuits, IEEE Journal of , vol.42, no.5, pp.954-966, May 2007

[2] Ingels, M.; Soens, C.; Craninckx, J.; Giannini, V.; Kim, T.; Debaillie, B.; Libois, M.; Goffioul, M.; Van Driessche, J., "A CMOS $100 \mathrm{MHz}$ to $6 \mathrm{GHz}$ software defined radio analog front-end with integrated pre-power amplifier," 33rd European Solid State Circuits Conference, 2007. ESSCIRC , vol., no., pp.436-439, 11-13 Sept. 2007

[3] Bagheri, R.; Mirzaei, A.; Chehrazi, S.; Heidari, M. E.; Lee, M.; Mikhemar, M.; Tang, W.; Abidi, A. A., "An 800-MHz-6-GHz Software-Defined Wireless Receiver in 90-nm CMOS," Solid-State Circuits, IEEE Journal of, vol.41, no.12, pp.2860-2876, Dec. 2006

[4] J. Crols, M. S. J. Steyaert, "Low-IF topologies for highperformance analog front ends of fully integrated receivers," IEEE Transactions on Circuits and Systems II, vol.45, no.3, pp.269-282, Mar 1998.

[5] Behbahani, F.; Firouzkouhi, H.; Chokkalingam, R.; Delshadpour, S.; Kheirkhahi, A.; Nariman, M.; Conta, M.; Bhatia, S., "A fully integrated low-IF CMOS GPS radio with on-chip analog image rejection," Solid-State Circuits, IEEE Journal of , vol.37, no.12, pp. 1721-1727, Dec 2002

[6] Jinho Ko; Jongmoon Kim; Sanghyun Cho; Kwyro Lee, "A 19-mW 2.6-mm/sup 2/ L1/L2 dual-band CMOS GPS receiver," Solid-State Circuits, IEEE Journal of, vol.40, no.7, pp. 1414-1425, July 2005

[7] Kai Borre, "The Galileo Signals With Emphasis on L1 OS," Power Electronics and Motion Control Conference, 2006. EPE-PEMC 2006. 12th International, vol., no., pp.2025-2030, Aug. 2006

[8] European Space Agency, "Galileo Open Service Signal In Space Interference Control Document (OS SIS ICD)", May 23, 2006.

[9] Valkama, M.; Renfors, M.; Koivunen, V., "Advanced methods for $\mathrm{I} / \mathrm{Q}$ imbalance compensation in communication receivers," Signal
Processing, IEEE Transactions on [see also Acoustics, Speech, and Signal Processing, IEEE Transactions on], vol.49, no.10, pp.23352344, Oct 2001

[10] M. Windisch, G. Fettweis, "Performance analysis for blind I/Q imbalance compensation in low-IF receivers," International Symp. on Control, Communications and Signal Processing, pp: 323-326, 2004

[11] Cetin, E.; Kale, I.; Morling, R.C.S., "Adaptive compensation of analog front-end I/Q mismatches in digital receivers," Circuits and Systems, 2001. ISCAS 2001. The 2001 IEEE International Symposium on, vol.4, no., pp.370-373 vol. 4, 6-9 May 2001

[12] Martin, K.W., "Complex signal processing is not complex," Circuits and Systems I: Regular Papers, IEEE Transactions on [Circuits and Systems I: Fundamental Theory and Applications, IEEE Transactions on], vol.51, no.9, pp. 1823-1836, Sept. 2004

[13] Mahattanakul, J., "The effect of I/Q imbalance and complex filter component mismatch in low-IF receivers," Circuits and Systems I: Regular Papers, IEEE Transactions on [Circuits and Systems I: Fundamental Theory and Applications, IEEE Transactions on] , vol.53, no.2, pp. 247-253, Feb. 2006

[14] Cetin, E., I. Kale and R. C. S. Morling, "Adaptive Self-Calibrating Image Rejection Receiver", IEEE International Conference on Communications (ICC 2004), vol. 5, pp. 2731-2735, June 2004

[15] Schmid, A., Neubauer, A., Ehm, H., Weigel, R., Lemke, N., Heinrichs, G., Winkel, J., Avila-Rodriguez, J. A., Kaniuth, R., Pany, T., Eissfeller, B., Rohmer, G., and Overbeck, M., "Combined Galileo/GPS architecture for enhanced sensitivity reception," International Journal of Electronics and Communications, Volume 59, Issue 5, pp. 297-306, July 2005. 